\title{
SLOTTED WAVEGUIDE SLOW-WAVE STOCHASTIC COOLING ARRAYS
}

\author{
D. McGinnis" ${ }^{\#}$ FNAL, Batavia, IL
}

\section{Abstract}

The slotted waveguide slow-wave stochastic cooling arrays are an integral part of the 4-8 GHz Debuncher Upgrade at FNAL. Unlike the standard array of stripline electrodes, these structures are designed to work when the beam pipe can support many microwave modes. The design theory and beam measurement results of this new type of pickup structure will be presented in this paper.

\section{INTRODUCTION}

In previous collider runs at Fermilab, all of the stochastic cooling pickup and kicker arrays consisted of stripline or planar loop electrodes. The signals from these electrodes are combined with a binary combiner tree formed by microstrip or stripline transmission lines. With a binary combining scheme, there must be no waveguide modes traveling down the beam pipe that would provide an alternate signal path in parallel to the binary combiner tree.

The nominal Debuncher transverse aperture is $30 \pi$ mm-mrad (95\% un-normalized). To account for closedorbit variations, the design aperture of the cooling arrays was set at $40 \pi$-mm-mrad. With lattice beta functions on the order of 10 meters, the transverse dimensions of the beam pipe will be about $40 \mathrm{~mm}$ which will propagate waveguide modes above $4 \mathrm{GHz}$. The presence of travelling waveguide modes in the beam pipe will limit the workable fractional bandwidth of the cooling arrays.

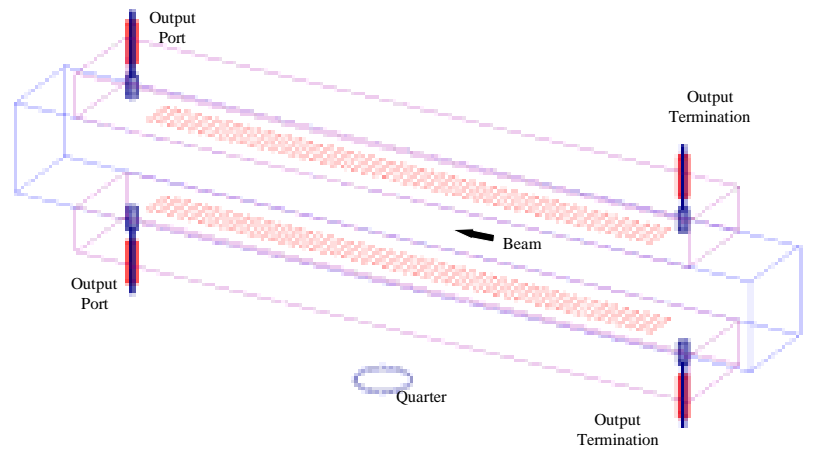

Figure 1: Conceptual drawing of a slow wave pickup

The solution was to divide the $4-8 \mathrm{GHz}$ bandwidth into 4 narrower bands with each band having a bandwidth of about $1 \mathrm{GHz}$. The cooling arrays are built with slot coupled "slow-wave" waveguide structures as

\footnotetext{
"Work supported by the United States Department of Energy under contract No. DE-AC02-76CH03000

"Email: mcginnis@fnal.gov
}

shown in Figure 1. The structure consists of two rectangular waveguides that are coupled to a rectangular beam pipe by a series of slots. The transverse signal is derived from the difference between the two waveguides and the momentum signal is derived from the sum of the two waveguides.

The image current that flows along the walls of the beam pipe due to a charged particle beam travelling in the center of the beam pipe excites electromagnetic magnetic waves in the slots which in turn excite travelling waveguide modes in the side waveguides and beampipe. Since the phase velocity of the unperturbed waveguide modes is faster than the beam velocity, the slots also act to "slow down" the waveguide modes by multiple reflections so that the phase velocity of the waveguide modes along the structure matches the beam velocity.

\section{MOMENT METHODS}

The structure can be analyzed using the moment method technique.[1] Using the equivalence principle[2], the slots in the sides of the waveguides can be replaced with metal walls and an equivalent magnetic current source as shown in Figure 2. The magnetic current source is derived from the tangential electric field in the slot. The magnetic field in Region I is:

$$
\overrightarrow{\mathrm{H}}_{\mathrm{t}}^{(1)}=\overrightarrow{\mathrm{H}}_{\mathrm{t}}^{(\mathrm{inc})}{ }_{1}+\vec{H}_{\mathrm{t}}^{(1)}\left(\overrightarrow{\mathrm{E}}_{\mathrm{t}} \times \hat{\mathrm{y}}\right)
$$

where the first term on the RHS of Eqn. 1 is the magnetic field as if the slot did not exist and the second term is the field due to the magnetic current source inside the slot. The magnetic field across the slot must be continuous:

$$
\overrightarrow{\mathrm{H}}_{\mathrm{t}}^{(\text {inc) }}{ }_{2}-\overrightarrow{\mathrm{H}}_{\mathrm{t}}^{(\text {inc })}=\vec{H}_{\mathrm{t}}^{(1)}\left(\overrightarrow{\mathrm{E}}_{\mathrm{t}} \times \hat{\mathrm{y}}\right)+\vec{H}_{\mathrm{t}}^{(2)}\left(\overrightarrow{\mathrm{E}}_{\mathrm{t}} \times \hat{\mathrm{y}}\right)
$$

This is the key equation of the moment method. Since $\mathrm{H}^{(\mathrm{inc})}$ is known, this equation can be inverted to determine $E_{t}$ in the aperture. Because Eqn. 2 is an integral equation, it is best solved by numerical methods. Let the tangential electric field in the aperture be given by:

$$
\overrightarrow{\mathrm{E}}_{\mathrm{t}}=\hat{\mathrm{x}} \sum_{\mathrm{n}} E_{\mathrm{x}_{\mathrm{n}}} \theta_{\mathrm{n}}(\mathrm{x}, \mathrm{z})+\hat{\mathrm{z}} \sum_{\mathrm{n}} E_{\mathrm{z}_{\mathrm{n}}} \psi_{\mathrm{n}}(\mathrm{x}, \mathrm{z})
$$

where $\theta_{\mathrm{n}}(\mathrm{x}, \mathrm{z})$ and $\psi_{\mathrm{n}}(\mathrm{x}, \mathrm{z})$ are a set of orthogonal functions. If the slots are very narrow in the $\mathrm{z}$ direction, the $\mathrm{x}$ component of electric field can be neglected. Equation 2 can be turned into a matrix equation by multiplying it by a set of orthogonal weighting functions $\phi_{\mathrm{m}}(\mathrm{x}, \mathrm{z})$ and integrating over the entire $\mathrm{x}-\mathrm{z}$ plane. 


$$
\begin{aligned}
& \left\langle\phi_{\mathrm{m}} \mid \mathrm{H}_{\mathrm{x}}^{(\mathrm{inc})}{ }_{1}\right\rangle-\left\langle\phi_{\mathrm{m}} \mid \mathrm{H}_{\mathrm{x}}^{(\mathrm{inc})} 2\right\rangle \\
& =\sum_{\mathrm{n}}\left(\left\langle\phi_{\mathrm{m}}\left|\boldsymbol{H}_{\mathrm{x}}^{(1)}\right| \psi_{\mathrm{n}}\right\rangle+\left\langle\phi_{\mathrm{m}}\left|\boldsymbol{H}_{\mathrm{x}}^{(2)}\right| \psi_{\mathrm{n}}\right\rangle\right) \mathrm{E}_{\mathrm{z}_{\mathrm{n}}}
\end{aligned}
$$

where:

$$
\left\langle\phi_{\mathrm{m}}\left|H_{\mathrm{v}}^{(\mathrm{k})}\right| \psi_{\mathrm{n}}\right\rangle=\iint_{\mathrm{x}, \mathrm{z}}\left(\phi_{\mathrm{m}}(\mathrm{x}, \mathrm{z}) H_{\mathrm{v}}^{(\mathrm{k})}\left(\hat{\mathrm{x}} \psi_{\mathrm{n}}(\mathrm{x}, \mathrm{z})\right)\right) \mathrm{dxdz}
$$

and:

$$
\left\langle\phi_{\mathrm{m}} \mid \mathrm{H}_{\mathrm{v}}^{(\mathrm{inc})} \mathrm{i}\right\rangle=\iint_{\mathrm{x}, \mathrm{z}}\left(\phi_{\mathrm{m}}(\mathrm{x}, \mathrm{z}) \mathrm{H}_{\mathrm{v}}^{(\mathrm{inc})}{ }_{\mathrm{i}}(\mathrm{x}, \mathrm{z})\right) \mathrm{dxdz}(6)
$$

Equation 4 forms a set of linear equations, which can be inverted to find the electric field coefficients $E_{\mathrm{Zn}}$. If the electric field expansion functions, $\phi$ and $\psi$, are chosen to be as close to the actual solution as possible then only a few terms of the expansion will be needed and the size of the matrix to be inverted will be minimized.

The electromagnetic fields leaving a region of sources as shown in Figure 3, can be expanded in terms of waveguide modes:

$$
\begin{gathered}
\overrightarrow{\mathrm{E}}^{ \pm}=\sum_{\mathrm{n}} \mathrm{C}_{\mathrm{n}}^{ \pm}\left(\hat{\mathrm{e}}_{\mathrm{t}_{\mathrm{n}}} \pm \hat{\mathrm{e}}_{\mathrm{z}_{\mathrm{n}}}\right) \mathrm{e}^{\mp j \beta_{\mathrm{n}} \mathrm{z}} \\
\overrightarrow{\mathrm{H}}^{ \pm}=\sum_{\mathrm{n}} \mathrm{C}_{\mathrm{n}}^{ \pm}\left( \pm \hat{\mathrm{h}}_{\mathrm{t}_{\mathrm{n}}}+\hat{\mathrm{h}}_{\mathrm{z}_{\mathrm{n}}}\right) \mathrm{e}^{\mp j \beta_{n} \mathrm{z}}
\end{gathered}
$$

where $e_{n}$ and $h_{n}$ are the waveguide modes. Using Lorentz reciprocity, the mode coefficients $C_{n}$ are given as [3]:

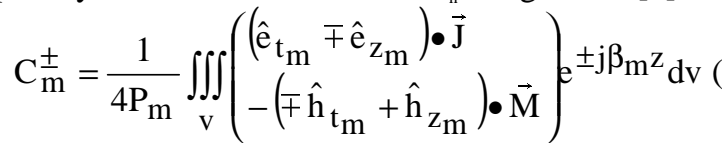

where

$$
\mathrm{P}_{\mathrm{m}}=\frac{1}{2} \iint_{\mathrm{S}_{\mathrm{t}}}\left(\hat{\mathrm{e}}_{\mathrm{t}_{\mathrm{m}}} \times \hat{\mathrm{h}}_{\mathrm{t}_{\mathrm{m}}}\right) \bullet \hat{\mathrm{z} d S}
$$

For example, the magnetic field in a waveguide due to a charged particle beam travelling in the $+\mathrm{z}$ direction located at $\mathrm{x}_{\mathrm{b}}, \mathrm{y}_{\mathrm{b}}$ :

$$
\vec{J}(x, y, z)=\hat{z} \frac{i_{b}}{2} \delta\left(x-x_{b}\right) \delta\left(y-y_{b}\right) e^{-j \kappa z}
$$

is given as:

$$
\begin{aligned}
& H_{x}^{\text {inc }}(x, y, z)= \\
& -\frac{i_{b}}{2} \sum_{n} \frac{e_{z_{n}}\left(x_{b}, y_{b}\right)}{4 P_{n}} \frac{2 j \beta_{n}}{\kappa^{2}-\beta_{n}^{2}} h_{x_{n}}(x, y) e^{-j \kappa z}
\end{aligned}
$$

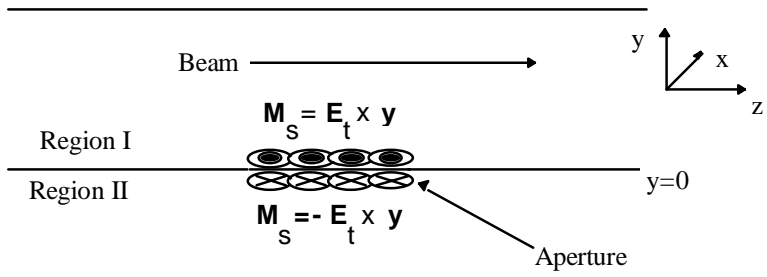

Figure 2: Equivalent magnetic source in slots.

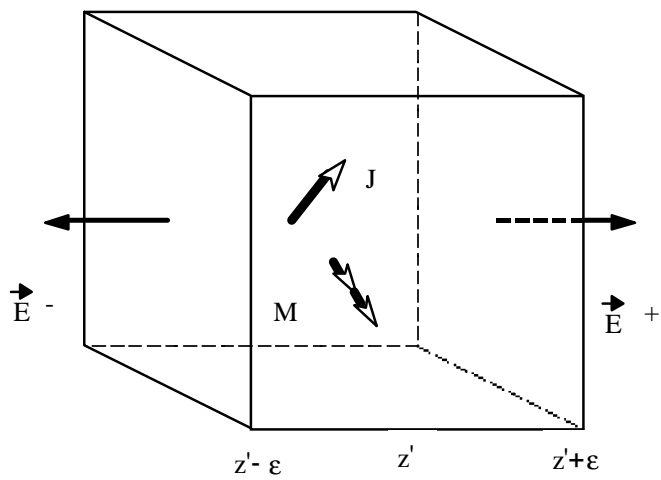

Figure 3: Elemental volume in waveguide containing sources

\section{ARRAY IMPEDANCE}

For the transverse mode, the pickup impedance of the array is derived from the relationship between the difference in power excited in opposing output waveguides as a function of beam current and beam position traveling through the array:

$$
\frac{1}{2} \mathrm{P}_{\Delta_{\text {total }}}=\frac{1}{2} \mathrm{Z}_{\Delta_{\mathrm{pu}}}\left(\frac{\mathrm{i}_{\mathrm{b}}}{2}\right)^{2}\left(\frac{\mathrm{y}}{\mathrm{d} / 2}\right)^{2}
$$

The kicker impedance of the array is derived from the relationship between the transverse change in beam momentum as a function of input power supplied to opposing input waveguides:

$$
\mathrm{P}_{\mathrm{k}}=\frac{1}{2} \frac{\left(\frac{\Delta \mathrm{pc}}{\mathrm{q}}\right)^{2}}{\mathrm{Z}_{\mathrm{k}}}
$$

where

$$
\frac{\Delta p c \mid}{q}=\int_{-\infty}^{\infty}\left(E_{y}-\eta H_{x}\right) e^{-j \kappa z} d z
$$

for a beam travelling in the $-\mathrm{z}$ direction. Since the ends of the array are not well defined, the limits of this integral are difficult to evaluate numerically.

Another way to evaluate the integral is to use Lorentz reciprocity on the geometry shown in Figure 4. The fields for the pickup case and the kicker case are related by:

$$
\begin{aligned}
& \oiint_{S_{\mathrm{O}}}\left(\overrightarrow{\mathrm{E}}^{\mathrm{p}} \times \overrightarrow{\mathrm{H}}^{\mathrm{k}}-\overrightarrow{\mathrm{E}}^{\mathrm{k}} \times \overrightarrow{\mathrm{H}}^{\mathrm{p}}\right) \bullet \hat{\mathrm{n}} \mathrm{d} S \\
& =\iiint_{\mathrm{v}}\left(\overrightarrow{\mathrm{H}}^{\mathrm{k}} \bullet \overrightarrow{\mathrm{M}}^{\mathrm{p}}-\overrightarrow{\mathrm{E}}^{\mathrm{k}} \bullet \overrightarrow{\mathrm{J}}^{\mathrm{p}}\right) \mathrm{dv}
\end{aligned}
$$

where the $\mathbf{k}$ indicates the kicker fields an sources and the $\mathbf{p}$ indicates pickup fields and sources. If the absorber kills the pickup and kicker fields at ports 2 and 4 and only the fundamental waveguide mode propagates in ports 1 and 3, then the left hand side of the reciprocity integral of Eqn. 14 is zero for ports 2, 3, and 4. The integral is non-zero only at port 1 . If a current distribution: 


$$
\begin{aligned}
\vec{J}^{p} & =\hat{y} \frac{i_{b}}{2} \delta\left(x-x_{b}\right) \delta\left(y-y_{b}\right) e^{-j \kappa z} \\
\vec{M}^{p} & =\hat{x} \eta \frac{i_{b}}{2} \delta\left(x-x_{b}\right) \delta\left(y-y_{b}\right) e^{-j \kappa z}
\end{aligned}
$$

(which is unphysical) is substituted into Eqn. 14 and a pickup impedance of the following form is defined:

$$
\tilde{\mathrm{P}}=\frac{1}{2}\left(\frac{\mathrm{i}_{\mathrm{b}}}{2}\right)^{2} \tilde{\mathrm{Z}}_{\Delta_{\mathrm{p}}}
$$

then the kicker impedance defined in Eqn 13 is given as:

$$
2 \tilde{Z}_{\Delta_{\mathrm{p}}}=\mathrm{Z}_{\Delta_{\mathrm{k}}}
$$

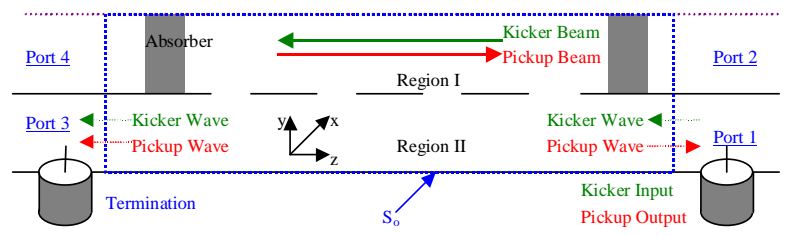

Figure 4: Slow wave array showing surface for reciprocity integral

\section{MEASUREMENTS}

A vertical slow wave pickup centered at $6 \mathrm{GHz}$ was installed in the Debuncher ring. The pickup consisted of 50 slots. The length of each slot was $17.5 \mathrm{~mm}$ and the width was $2 \mathrm{~mm}$. The metal spacing between each slots was $3 \mathrm{~mm}$. The height of the output waveguide was 20 $\mathrm{mm}$ and the width was $40 \mathrm{~mm}$. The width and the height of the beam pipe were $40 \mathrm{~mm}$.

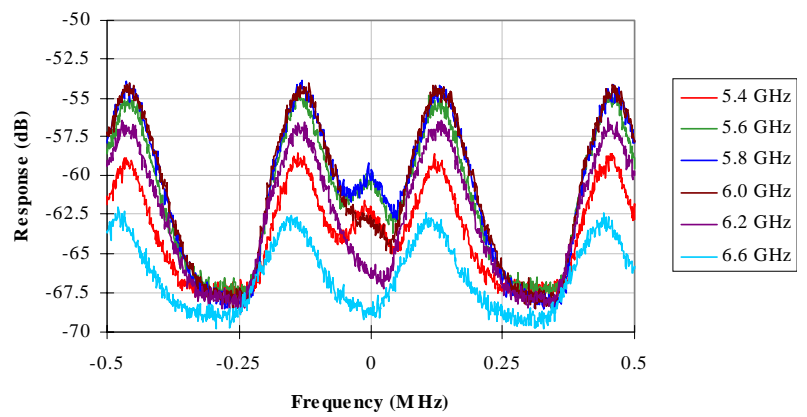

Figure 5. Narrow-band signal of the slow wave pickup in the difference mode at several microwave frequencies

Unbunched, coasting beam was injected into the Debuncher and stored. The beam was transversely excited with RF noise until the beam filled the transverse aperture. The power spectrum was measured with a spectrum analyzer at several frequencies as shown in Figure 5. . The transverse aperture could be varied by means of a mechanical beam-scraping device (scraper). The transverse beam distribution was determined by measuring the amount of beam current remaining as a function of scraper position.

The impedance of the array was determined by measuring the power spectral density with a spectrum analyzer with and without beam. The resolution bandwidth of the spectrum analyzer was set much greater than the revolution frequency of the Debuncher so as to average over the momentum distribution of the beam. The sum mode impedance is:

$$
\mathrm{Z}_{\Sigma}=(\mathrm{g}-1) \frac{\mathrm{N}_{\mathrm{f}} \mathrm{S}_{\text {therm. }}}{\mathrm{eI} \mathrm{I}_{\mathrm{dc}}}
$$

The difference mode impedance is

$$
\mathrm{Z}_{\Delta}=(\mathrm{g}-1) \frac{\mathrm{N}_{\mathrm{f}} \mathrm{S}_{\text {therm. }}}{2 \mathrm{eI}_{\mathrm{dc}}\left(\frac{\sigma}{\mathrm{d}}\right)^{2}}
$$

where $\mathrm{N}_{\mathrm{f}}$ is the noise figure of the pre-amplifier, $\mathrm{S}_{\text {therm }}$ is the power spectral density of white thermal noise (which is equal to $-174 \mathrm{dBm} / \mathrm{Hz}$ at room temperature), $I_{d c}$ is the beam current, $\sigma$ is the r.m.s transverse size of the beam, and $d$ is the transverse size of the beam pipe. The quantity " $\mathrm{g}$ " is defined to be the ratio of the power per revolution band when there is beam in the machine to when there is no beam in the machine:

$$
\mathrm{g}=\frac{\mathrm{P}_{\text {beam }}+\mathrm{P}_{\text {noise }}}{\mathrm{P}_{\text {noise }}}
$$

The results are shown in Figures 6 and 7. The calculated impedance had to be adjusted by $4 \mathrm{~dB}$ to account for the measured cable and hybrid loss between the array and pre-amplifier.

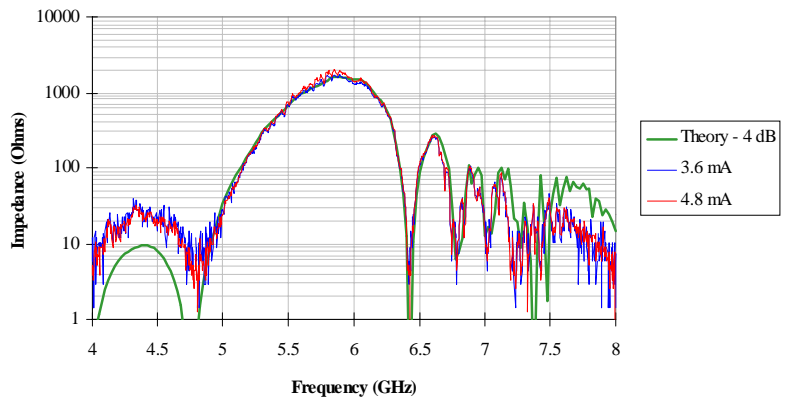

Figure 6. Difference response of the slow wave pickup.

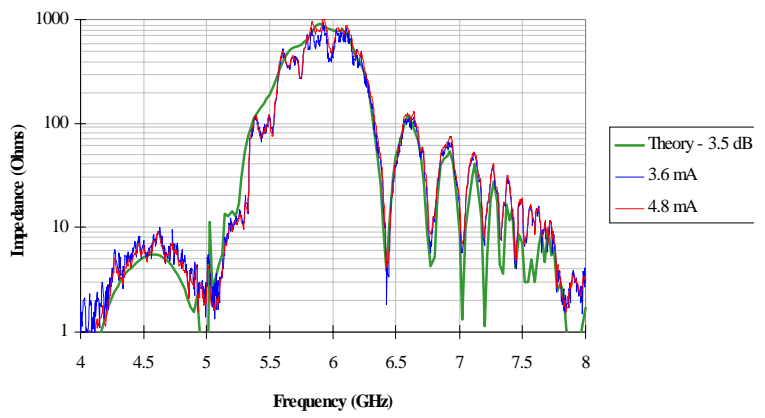

Figure 7. Sum response of the slow wave pickup.

\section{REFERENCES}

[1] R.F. Harrington, "Matrix Methods for Field Problems," Proceedings of the IEEE,Vol. 55, No.2, February 1967, pg 136-149

[2] R. F. Harrington, Time Harmonic Electromagnetic Fields, McGraw Hill, 1961, pg 106-109

[3] R.E. Collin, Foundations for Microwave Engineering, McGraw-Hill, 1966 pg 184-185. 